

\section{AUTONOMÍA Y NECESIDADES BÁSICAS}

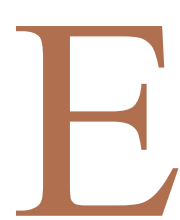

1 reconocimiento de necesidades básicas como dato relevante para distribuir bienes, disponer tratamientos, asignar derechos y obligaciones parece dividir aguas entre diversas concepciones de filosofía política. Mientras que los enfoques que parten de una visión teleológica de la naturaleza humana, como el aristotélico -tomista- o el marxismo asumen en forma central ese reconocimiento, el liberalismo, en sus diferentes versiones, parece excluir el reconocimiento de necesidades básicas en favor del lugar central que ocupan los deseos o preferencia en los criterios de distribución propuestos.

El objeto de este artículo es reivindicar el papel relevante del reconocimiento de necesidades básicas en el marco de una concepción liberal de la sociedad.

1. Ante todo es conveniente hacer ciertas aclaraciones sobre el concepto de necesidad básica. Wiggins ${ }^{1}$ contesta al economista que le preguntó si una necesidad no es al fin y al cabo un deseo por el que uno no está dispuesto a pagar, diciendo que una necesidad se distingue en forma relevante de los deseos: mientras yo puedo desear comer un plato de ostras, pero no las ostras envenenadas que hay en el plato, el necesitar algo no es intencional, no depende del estado mental del agente, sino de la realidad. Si bien hay un sentido instrumental de necesidad que depende de los deseos del agente, hay un sentido absoluto en que el fin al que la necesidad está condicionada está fijado como parte del concepto. Ese fin es el de evitar un daño que está definido por normas, relativas a ciertas circunstancias, de florecimiento humano. Este tema es retomado por Frankfurt ${ }^{2}$ cuando explica el

${ }^{1}$ Ver David Wiggins, «Claims of Need», en Morality and Objectivity, T. Honderich, comp., Londres, 1985, pág. 152.

${ }^{2}$ Ver Harry Frankfurt, «Necessity and Desire»... 
principio de precedencia de las necesidades sobre los deseos sobre la base de que hay necesidades «categóricas» que no dependen de deseos y que no se puede evitar tener. La privación de estas necesidades constituye un daño que debe distinguirse de la mera ausencia de beneficio que se produce cuando se frustra un deseo. La precedencia de las necesidades sobre los deseos se funda en el hecho de que es peor causar un daño que no dar un beneficio.

Sin embargo, este criterio es objetado en forma convincente por Goowin, ${ }^{3}$ quien sostiene que la distinción entre causar un daño y no dar un beneficio se debe al hecho contingente moralmente de si el individuo tenía acceso previamente al bien en cuestión, en cuyo caso su privación constituye un daño, o no lo tenía, en cuyo caso consiste en la mera negación de un beneficio. Por lo tanto, el principio de precedencia de las necesidades sobre los deseos tal como es interpretado por Frankfurt implícitamente favorece el mantenimiento del statu quo.

Goowin examina también la posibilidad de defender la precedencia de necesidades sobre deseos sobre la base del principio liberal de autonomía personal. Las necesidades estarían supeditadas al fin de promover la autonomía de la persona. Pero esta posición parece paradójica porque conduce a frustrar deseos previamente formados para proteger la formación de otros deseos. La creación de autonomía no puede ser más importante que el ejercicio de esa autonomía, sino que a lo sumo debe haber un compromiso entre estos dos objetivos.

Aquí llegamos al punto crucial para determinar el papel de las necesidades en el marco de una concepción liberal de la sociedad: las necesidades cuyo reconocimiento es relevante analizar son las categóricas o absolutas, o sea, aquellas que están supeditadas a fines que no dependen de los deseos o preferencias de los agentes. Dado que el valor básico de una concepción liberal de la sociedad es la autonomía personal, esas necesidades deberían identificarse como estados de cosas que son prerrequisitos de esa autonomía. Pero la autonomía personal, como seña la Goowin, tiene dos caras: su creación y su ejercicio. La primera no depende de los deseos y preferencias de la gente, mientras que la segunda sí depende. Para que las necesidades categóricas tengan un lugar central en una concepción liberal de la sociedad la creación de autonomía debe tener preeminencia sobre su ejercicio. Veamos esta cuestión con más cuidado.

${ }^{3}$ Ver Robert E. Goowin, «The Priority of Needs», en Philosophy and Phenomenological Research, 45, 1985. 
2. Conviene repasar primero brevemente cuál es el alcance y fundamento del principio de autonomía personal en una concepción liberal de la sociedad. Según mi interpretación favorita, ${ }^{4}$ la práctica social del discurso moral en cuyo contexto se cuestionan principios y posiciones valorativas como la que estamos discutiendo está dirigida a obtener consenso como una forma de superar conflictos y facilitar la cooperación mediante la libre aceptación compartida de principios que permitan converger en acciones y actitudes. La idea de consenso incluye la noción amplia de autonomía moral que está dada por la libre aceptación de principios para guiar la propia conducta. Esto significa que la participación genuina y honesta en el discurso presupone la aceptación del valor de esa autonomía moral y que se incurre al menos en alguna inconsistencia práctica cuando se participa en el discurso moral para defender algún principio que sea incompatible con ese valor. Pero el valor de la autonomía moral juega en forma diferente según el tipo de principios a cuya libre aceptación se refiere: cuando se trata de principios intersubjetivos que juzgan una conducta por los efectos que ella tiene en los intereses de otros, el valor positivo de la aceptación libre de un principio de esta clase puede verse neutralizado por el valor negativo que tiene esa aceptación si el principio justifica conductas que afectan a la autonomía de otra gente. De este modo, el mismo valor de autonomía moral puede justificar coartar la libre elección de principios de conducta cuando ellos se refieren a relaciones interpersonales (cómo se hace un balance entre la autonomía del agente que se limita y la autonomía de otros individuos que resulta promovida depende de principios de distribución de autonomía como los que he denominado los principios de inviolabilidad y dignidad de la persona). ${ }^{5}$ Pero lo mismo no ocurre cuando se trata de principios morales autorreferentes que juzgan una conducta por los efectos que ella tiene en el carácter o en la vida del propio agente. En este caso el valor de la autonomía moral no puede justificar una restricción de tal autonomía moral (salvo el caso del paternalismo perfeccionista que merece un tratamiento aparte), ${ }^{6}$ además de que generalmente resulta autofrustrante imponer principios autorreferentes -ideales del bien personal y planes de vida basados en ellos-, ya que ellos incluyen como componente esencial su adopción espontánea. De este modo, del principio general de autonomía moral

\footnotetext{
${ }^{4}$ Ver Ética y derechos humanos, 2. ${ }^{\text {a }}$ ed., Buenos Aires, 1989, cap. 4.

${ }^{5}$ Ver op cit., en nota ant., caps. 5 y 6.

${ }^{6}$ Ver op. cit., en nota 4, cap. 4.
} 
que está presupuesto en el discurso moral se deriva el principio más específico de autonomía personal que valora la libre elección y materialización de ideales del bien y de virtud personal.

Pero en la última formulación ya emergen las dos caras de la autonomía personal, puesto que es necesario tomar en cuenta tanto la elección como la materialización de concepciones y planes de vida. A primera vista esta oposición parece falsa, ya que cada uno de estos aspectos no tiene valor sin el otro: no tiene sentido elegir planes de vida que no se pueden materializar y no tiene valor, en el contexto de una concepción liberal de la sociedad, materializar planes de vida que uno no ha elegido libremente.

Sin embargo, la oposición es real y se pone de manifiesto tan pronto tenemos en cuenta el dato de la escasez de bienes. Un conjunto finito de bienes puede ser comparado con otro teniendo en cuenta hasta qué punto nos capacitan para elegir planes de vida materializables. También pueden ser comparados teniendo en cuenta hasta qué punto nos permiten satisfacer un cierto plan de vida libremente elegido. El conjunto de bienes que resulta superior en la primera comparación no necesariamente resulta superior cuando hacemos la segunda. Por ejemplo, poseer grados aceptables de educación y formación cultural, buen estado físico, medios económicos decorosos permite un amplio menú de planes de vida, pero quien tiene una superlativa educación y entrenamiento intelectual, aun a costa de su estado físico y de sus medios económicos, puede satisfacer en mayor medida su ambición de dedicarse a la filosofía que quien goza de aquella variedad de recursos moderados.

Esta oposición se da tanto intrasubjetivamente como intersubjetivamente. El enriquecimiento de un individuo en un sentido -sea el de la creación o el del ejercicio de la autonomía- puede implicar su empobrecimiento en el otro. Y la ampliación de las opciones de otros individuos puede provocar que uno tenga menos posibilidades de satisfacer el plan de vida elegido (resintiendo el desperdicio de recursos que otros no usan, pero podrían usar, y que uno aplicaría al proyecto de vida favorecido).

Por supuesto que, dependiendo del dato de la escasez de bienes, esta oposición se pone sólo de manifiesto cuando se encara no simplemente la cuestión del valor de la autonomía, sino la de su distribución. No hay duda que el valor de autonomía implica el valor de la libre elección de planes de vida materializables. El problema es si una distribución igualitaria de ese valor debe operar en la dimensión de la libre elección o en la de la materialización. 
3. Frente a la acusación de que centrar el problema de la distribución de acuerdo a su principio de diferencia en los bienes primarios es una posición fetichista, ya que nadie valora a los bienes primarios de por sí, sino por la utilidad o satisfacción que prestan, Rawls ${ }^{7}$ ha sostenido que basarse en tales bienes primarios es la única manera de hacer a los individuos responsables de sus deseos o preferencias de tal modo que los adopten o no de acuerdo a los recursos disponibles para satisfacerlos. De otro modo, los deseos y preferencias serían tratados como meros hándicaps que hay que indemnizar y a los individuos como meros pacientes de tales deseos y preferencias. Para Rawls ellos no constituyen razones que justifiquen acciones o medidas en el área de la justicia. Reconoce, sin embargo, este autor que su posición exige un tratamiento especial de los verdaderos hándicaps que hay que indemnizar no obstante que no puede darse la razón de que los minusválidos sacan menor utilidad de la misma cantidad de recursos.

En su conocido artículo «Preference and Urgency», ${ }^{8}$ Scanlon adopta la misma posición de defender un criterio objetivo sobre la urgencia de ciertas necesidades que no depende de la intensidad de los deseos o preferencias de los individuos. «Mi tesis -sostienees que cuando nos proponemos comparar intereses conflictivos con el objetivo de apoyar un juicio moral acerca de cuál interés debe prevalecer, lo que hacemos no es comparar cuán fuertemente la gente siente acerca de esta cuestión (tal como se determina tal vez por lo que ellas están dispuestas a sacrificar para alcanzar lo que quieren), sino inquirimos acerca de las razones por las cuales tales beneficios son considerados deseables.» De lo contrario la gente con gustos tremendamente caros debería absorber buena parte de los recursos que otros individuos usarían para satisfacer en más alto grado preferencias más modestas.

Pero quien ha analizado con más profundidad la oposición entre lo que él llama «igualdad de bienestar» e «igualdad de recursos» es Ronald Dworkin en su extenso artículo «What is Equality?» ${ }^{9}$ Allí este autor despliega una artillería extremadamente

${ }^{7}$ Ver principalmente su artículo «Social Unity and Primary Goods», en Utilitarianism and Beyond, A. Sen y B. Williams comps., Cambridge, 1982.

${ }^{8}$ En The Journal of Philosophy, vol. LXXXIII, núm. 19, 1975.

${ }^{9}$ «Part 1: Equality of Welfare», Philosophy and Public Affairs, vol. 10, núm. 3, 1981, y «Part 2: Equality of Resources», la misma publicación, vol. 10, núm. 4, 1981. 
destructiva en contra de la idea de igualar los niveles de bienestar que la gente puede lograr. Para eso distingue varias teorías del bienestar -del «éxito», basadas en el goce, objetivas, etcétera-. En todos los casos excluye como fuente de bienestar, o sea, como objeto de preferencias o fuente de goce, cuestiones políticas o impersonales, ya que es absurdo que alguien sea compensado por la frustración de sus preferencias o goce, por que la sociedad no adoptó cierto esquema político o cierto valor impersonal por considerarlo incorrecto. Pero aun limitada a las cuestiones personales la teoría del bienestar es defectuosa porque la gente puede asignar diferente valor a la satisfacción de preferencias o al goce y no parece atractivo igualar a la gente en algo que valora desigualmente o compensar a los individuos que va loran menos que otros la satisfacción de preferencias o el placer. Estas teorías deben presuponer algún criterio objetivo como el que toma en cuenta cuando sería razonable lamentarse por la frustración de preferencias, pero estos criterios presuponen algún principio de distribución de recursos.

En cuanto se refiere a tal principio de igualdad en la distribución de recursos, Dworkin hace avances sumamente esclarecedores sobre la idea de los bienes primarios de Rawls, como, centralmente, la sugerencia de que la noción de un mercado ideal y de un seguro hipotético es un presupuesto conceptual de la igualdad de recursos. Esto permite encarar más eficazmente cuestiones como la del desperdicio de los recursos no usados para la satisfacción de planes de vida elegidos o la de la compensación de deficiencias físicas o intelectuales. Quizá uno podría avanzar aún más en esta línea de articulación de este principio de igualdad si, como lo propone A. $\operatorname{Sen}^{10}$ reemplazamos la noción de recursos por algo intermedio entre ellos y la utilidad o el bienestar, que recoge la idea de que los recursos pueden tener diferente valor para los individuos sin que ello dependa de sus gustos o preferencias: por ejemplo, igual cantidad de comida puede tener diferente poder nutricional para distintos individuos. Por eso Sen prefiere poner como objeto de la distribución igualitaria las capacidades («capabilities») que están determinadas por el funcionamiento de los individuos.

Pero a pesar de este esclarecimiento conceptual, es notable la falta de argumentos en apoyo de la defensa que estos autores hacen de la igualdad de bienes primarios, recursos o capacidades frente a la igualdad de bienestar o de utilidad, como es explícito

\footnotetext{
${ }^{10}$ Ver «Capability and Rights», en la colección citada en nota 1.
} 
en el caso de Dworkin cuando advierte que no se propone fundamentar el principio defendido.

El único argumento recurrente en el que estos autores apoyan su predilección por la creación de autonomía sobre su ejercicio, a los efectos de una distribución igualitaria, es el de que los hombres deben hacerse responsables por las consecuencias de sus deseos o preferencias, las que no deben ser tratadas como accidentes o deficiencias. Pero este argumento es insuficiente por dos razones: la primera es que el principio liberal que da relevancia moral al consentimiento en contra del determinismo normativo -principio que yo llamo «de dignidad de la persona»- permite tomar a ciertos actos expresivos de consentimiento como antecedente de cargas y responsabilidades, pero no obliga a hacerlo, ya que no hay ningún valor positivo que se satisface por el hecho de que cada acto voluntario de los individuos acarree consecuencias. En segundo lugar, y conectado con lo anterior, las consecuencias que se atribuyen a ciertas decisiones o actos voluntarios que son expresión de consentimiento son, en primer lugar, consecuencias normativas que ciertas prácticas sociales o reglas jurídicas atribuyen a las decisiones o actos en cuestión aprovechando la permisión moral de hacerlo y en persecución de algún valor substantivo como es la maximización de autonomía. Las consecuencias fácticas que se siguen de ciertas decisiones son supervinientes a estas consecuencias normativas que hay que justificar valorativamente. Por cierto que en este caso no podemos recurrir al valor de autonomía para justificar atribuir a las decisiones de los individuos consecuencias normativas tales que produzcan diferentes niveles de satisfacción de acuerdo al costo social de los recursos, ya que de lo que se está tratando es precisamente de interpretar el valor de autonomía en el marco de un principio de distribución.

4. Creo que la clave para comenzar a completar el argumento anterior se encuentra en la referencia de Rawls al hecho de que los deseos y preferencias no constituyen razones justificatorias de acciones y medidas en el área de la justicia. Pero esto debe verse con especial cuidado. El liberalismo ocupa una posición intermedia e inestable entre dos teorías opuestas que él rechaza: por un lado, la visión perfeccionista de acuerdo a la cual los deseos o preferencias personales no constituyen razón alguna para justificar acciones o medidas y que las únicas razones justificatorias de tales medidas o acciones son las que válidamente, según una cierta concepción verdadera del bien, fundamentan ciertas preferencias personales. La posición opuesta es la utilitarista 
que adopta una concepción del bien según la cual hay razones para satisfacer preferencias personales, cualquiera sea su contenido, y tomando en cuenta su alcance e intensidad. De acuerdo a la primera teoría, una preferencia, por, supongamos, hacer un costoso monumento a un dios no tiene ningún título a ser satisfecha si la concepción del bien asumida como válida condena a ese acto -tal vez por consideraciones teológicas- como autodegradante. Según la segunda teoría, esa preferencia tiene tanto título a ser satisfecha como la que se refiere a la comida necesaria para subsistir si el individuo que detenta la primera lo hace con la misma intensidad que el que profesa la segunda.

La posición intermedia del liberalismo parte de asignar un tratamiento opuesto a las preferencias impersonales y políticas, por un lado, y a las preferencias personales por el otro. En cuanto a las primeras deben ser objeto de discusión y decisión pública, presuntamente a través del procedimiento democrático, y sólo en la medida en que, por este medio, ${ }^{11}$ se determine su validez deben ser objeto de satisfacción de acuerdo a su contenido y a las razones que apoyan la preferencia en cuestión. En relación a las preferencias personales su reconocimiento como válidas o no, no debe ser objeto de decisión pública y, sin embargo, debe permitirse a sus titulares alcanzar algún grado de satisfacción con independencia de su validez y, por consiguiente, de las razones que las fundamentan.

La forma en que el liberalismo puede obtener el difícil equilibrio entre el perfeccionismo y el utilitarismo es admitiendo que esas preferencias, para ser atendibles, deben presuponer una concepción del bien que incluye a la autonomía como elemento central. Ahora bien, toda concepción del bien que incorpora el valor de la autonomía personal sólo provee razones relativas para actuar: razones que tienen un diferente impacto en la justificación de acciones según se trate de la persona cuya vida o carácter es valorada por la concepción en cuestión o de terceros. El principio de autonomía personal provee razones que tienen una relatividad peculiar: Si las razones que subyacen a preferencias personales no son válidas, ni el agente ni terceros están justificados en principio a actuar o a ayudar a actuar para satisfacer esas preferencias. Sin embargo, si el agente cree que esas razones son válidas ello, si bien no justifica su propio comportamiento,

\footnotetext{
${ }^{11}$ Ver op. cit., en nota 4, cap. 8, una justificación de la democracia que justifica este tratamiento de las preferencias impersonales.
} 
sí justifica las acciones de terceros tendentes a ayudarlo a actuar de acuerdo a sus falsas creencias. ¿Cómo puede ser que lo que no es razón para el agente sea una razón para los terceros?

Supongamos que sea parte de la concepción del individuo sobre lo que da valor a su vida una cierta visión religiosa que exige determinadas actitudes y comportamientos. Por supuesto que hasta el mismo individuo de que hablamos admitiría que si su visión religiosa fuera falsa sus acciones no estarían justificadas. Para el individuo en cuestión el hecho de que él crea que su concepción religiosa es correcta no cambia la conclusión de que si él estuviera equivocado no tendría razones para justificar sus acciones y actitudes. En cambio, parece que el valor de la autonomía personal implica que los terceros sí estarían justificados a ayudar al individuo a materializar su visión religiosa sobre la base del hecho de que él la sustenta y con independencia de que sea verdadera o falsa.

Si la visión religiosa del individuo fuera verdadera, por su puesto que ciertas acciones y actitudes de su parte estarían justificadas. En cierto sentido, ello es independiente del hecho de que el sujeto crea en esa visión, ya que su validez implica precisa mente la exigencia de que el individuo crea en ella y de que actúe en consecuencia. Pero, en otro sentido, las acciones y actitudes del individuo no serían correctas aun cuando se conformaran con la visión religiosa válida si el individuo no cree en esa validez, ya que en ese caso serían el resultado de la hipocresía o del temor y no realzarían el valor de su vida.

Desde el punto de vista de los terceros esta última condición de que el individuo crea en la visión religiosa válida es necesaria para justificar que lo ayudemos en su materialización bajo el valor de la autonomía personal. Si tal condición no se diera, el ayudarlo a satisfacer la visión religiosa implicaría cancelar su autonomía y conducirlo a realizar acciones que, como vimos, serían incorrectas aun cuando la concepción en cuestión fuera válida.

Esto parece indicar que, mientras para el individuo de que se trate es la validez de las razones subyacentes a sus preferencias personales y no su creencia en tal validez -aunque tal creencia sea condición necesaria de la corrección de su conducta-, lo que justifica sus acciones, para los terceros la justificación para actuar está dada por el hecho de que los interesados crean en ciertas razones que fundamentan sus preferencias personales -lo que es condición necesaria y suficiente para justificar la acción de esos terceros- y no depende en nada de la validez de tales razones. 
De aquí aparentemente se sigue que la autonomía proporciona razones relativas para actuar en este sentido: mientras para los interesados es el aspecto interno de las preferencias, o sea, la concepción del bien subyacente a ellas en el caso de ser válida, para los terceros es el aspecto externo de las preferencias -el hecho de que el individuo involucrado las tiene- lo que provee esas razones.

Esto parece justificar que la acción de terceros como el Esta do no varíe según sea el contenido y alcance de las preferencias, que dependen de las presuntas razones que subyacen a ellas. Si lo único que debe tomar en cuenta el Estado en sus medidas y acciones es el aspecto externo de las preferencias, tales medidas no deben variar sino en función de ese aspecto externo, por ejemplo, tomando en cuenta la intensidad con que se tiene la preferencia. Esto implicaría que la acción estatal al ser ciega respecto de la validez de la concepción del bien que subyace a las preferencias debe ser insensible al costo de su satisfacción y dis tribuir recursos tomando sólo en cuenta el hecho de que los in dividuos tienen preferencias de cierta intensidad. Dado que todos los individuos normales tienen preferencias de grados equivalentes de intensidad -aunque su contenido y alcance varíen de unos a otros- la consecuencia parecería ser que los recursos deben ser distribuidos igualitariamente.

Sin embargo, esta explicación, que según creo está bien orientada, es insuficiente. Los hechos en sí mismos no pueden proveer razones para justificar acciones o decisiones. No obstante que podría sostenerse que los hechos constituidos por el aspecto externo de deseos o preferencias son operativos en virtud del principio de autonomía de la persona, esto no da una explicación de por qué son esos hechos, y no, por ejemplo, los constituidos por la frustración de deseos o preferencias ya formados, los que ese principio convierte en operativos para justificar medidas o acciones. Me parece que una explicación más completa inevitablemente debe profundizar la articulación del valor de la autonomía personal.

5. Veamos qué concepción de la autonomía está involucrada en el enfoque que prescribe que los individuos deben ser tratados igualitariamente en la dimensión que toma en cuenta el resultado del ejercicio de la autonomía. Dado que, de acuerdo a este enfoque, los individuos tendrán igual grado de satisfacción cualquiera sea el plan de vida elegido (lo que variará equitativa mente no sólo por la elección de los individuos, sino también por la de todos los demás), lo que se promueve es el ejercicio de 
la imaginación de cada uno sobre cuál sería su plan de vida preferible con independencia de los recursos disponibles. El objeto de valoración es el acto mental de elegir un plan de vida y se pretende proteger esa elección prometiendo al individuo que, cualquiera que ella sea, él alcanzará igual grado de satisfacción. Lo que es propio del individuo es el acto de elección de un cierto plan de vida; una vez que se da esa elección la concreción del plan de vida es algo que involucra a todos, al comprometer la contribución de todos en la provisión de los recursos para que el individuo alcance el mismo grado de éxito o de goce que el resto.

En cambio, en el enfoque que prescribe igualar a los individuos en la dimensión que toma en cuenta las condiciones para elegir planes de vida materializables, lo que se valora no es el acto mental de elección, sino la creatividad del individuo al plasmar su vida de una forma u otra con los recursos disponibles. Esta articulación de la autonomía, que es la que resulta prima facie atractiva en contraste con las dudas que despierta el valor de la mera elección con abstracción de los recursos disponibles, requiere poner a la autonomía en el contexto de una concepción del bien más amplia.

Creo que esa concepción no es otra que la vieja noción de la auto-realización, ${ }^{12}$ que se relaciona con la alusión que hace Frankfurt al florecimiento y que ha sido muchas veces desvirtuada hacia direcciones perfeccionistas por no advertir que ella no es equivalente a realización personal sino a realización autónoma, o sea, a una realización de la que es autor el propio individuo concernido. En el centro de esta concepción del bien personal, está la idea de capacidades rescatada ahora por Amartya Sen. El individuo se auto-realiza en la medida en que actualiza en forma plena y equilibrada sus diversas capacidades. Hay una indefinida variedad de alternativas de desarrollar en plenitud algunas de las capacidades sin obliterar completamente las restantes y lo que se valora es la creatividad de los individuos en la exploración de estas alternativas. Por supuesto, uno de los parámetros que se toman en cuenta para evaluar esa creatividad es el grado de satisfacción de preferencias o de goce que el individuo obtiene con el desarrollo de algunas de sus capacidades. En esta concepción lo que es propio de cada individuo es el curso que da a su vida ejercitando con sus diversos actos una u otra capacidad, mientras que los demás están involucrados en la conformación de sus capacidades iniciales.

\footnotetext{
${ }^{12}$ Ver este punto en mi artículo «Liberalismo versus comunitarismo», en Revista del Centro de Estudios Constitucionales, Madrid, 1988.
} 
Esta articulación todavía bastante impresionista del valor de autonomía en el marco de una concepción más amplia del bien personal sugiere que, efectivamente, debemos igualar a los individuos en la dimensión de sus capacidades, lo que implica satisfacer ciertas necesidades básicas. Esto permite, efectivamente, asignar un lugar central a las necesidades categóricas en una concepción liberal de la sociedad, ya que la distribución pública de recursos debe atender a los prerrequisitos para la formación libre de preferencias satisfacibles en algún grado y no tomar en cuenta la satisfacción de preferencias libremente formadas en algún grado. Las necesidades básicas están sólo condicionadas por la razón proporcionada por el principio de autonomía personal que, por supuesto, no depende de las preferencias de los individuos. Sólo de este modo promovemos la creatividad de los individuos en la plasmación de su vida, que no es un mero ejercicio inicial de la imaginación, sino que consiste en una virtud que se manifiesta en todas las opciones que hacemos en el curso de nuestra vida, dadas ciertas condiciones preexistentes que no se van adaptando a cada una de esas decisiones.

También esta articulación implica confirmar que la autonomía es parte de una concepción más amplia del bien personal y que, en consecuencia, cuando promovemos a aquélla no estamos siendo neutrales frente a diversas concepciones del bien. Lo que ocurre es que precisamente por ser la autonomía un componente central del bien de la auto-realización el reconocimiento de este bien tiene un impacto diferente en el razonamiento práctico del agente y de terceros, proveyendo, como vimos antes, razones relativas. La relatividad de las razones que provee el bien de la auto-realización se expresa en términos que aluden a las necesidades básicas: mientras que los terceros, incluyendo el Estado, deben atender a la satisfacción de las necesidades de los individuos, o sea, a una maximización igualitaria de sus capacidades, sólo compete a cada individuo el ejercicio de esas capacidades en cualquiera de las múltiples alternativas que ellas ofrecen (por supuesto siempre que no afecten a igualdad en las capacidades de que gozan otros individuos). Si bien el ejercicio que los individuos puedan hacer de sus capacidades resulta muchas veces insuficiente o desequilibrado, cualquier intervención de terceros para ayudarlo a superar esas deficiencias, frustraría el bien de la auto-realización.

Sin embargo, es claro que hay una precedencia y no una exclusión de las necesidades sobre las preferencias en la distribución de recursos o capacidades: toda versión del liberalismo asigna un lugar importante a las transacciones que los individuos 
puedan hacer, para satisfacer en más alto grado las preferencias adoptadas, con los recursos sobrantes que no son necesarios para esa satisfacción, pero que le fueron asignados como prerrequisitos para ampliar su menú de preferencias. Esto es así sea porque un mercado ideal o real es un presupuesto conceptual de la idea de igualdad de recursos -como en el caso de Dworkin-, o sea, porque el principio de dignidad de la persona permite tomar en cuenta el consentimiento en el marco de instituciones como el mercado que pueden ser instrumentalmente adecuadas para maximizar el valor de autonomía igualitaria mente distribuida.

6. Hay, no obstante, una consideración adicional para que las necesidades básicas tengan un papel prioritario en una concepción liberal de la sociedad. Si se adoptara el principio de igualdad en el ejercicio de la autonomía, o sea, en el grado de satisfacción de preferencias personales, cada individuo sufriría en su propia vida el impacto de las preferencias personales de otros individuos. Un individuo, por ejemplo, podría decidir ser poeta tomando en cuenta que, dada la configuración de preferencias existentes en su sociedad, tendrá un grado $x$ de satisfacción; luego ocurre que una gran cantidad de individuos adoptan preferencias muy costosas, por ejemplo, la de ser astronautas, que baja el grado general de satisfacción a $x$ - $n$. Dado que la vida de cada individuo resultaría afectada no sólo por sus propias preferencias personales, sino también por la de los demás, se desvanece la idea de independencia y separabilidad de los individuos que subyace al principio de inviolabilidad de la persona. Esta idea asume que las preferencias personales tienen un ámbito de validez personal que está dado por la vida de cada individuo.

El presupuesto liberal que conduce al principio de inviolabilidad de la persona es que la vida de los individuos no debe ser afectada por decisiones de otros salvo que estén fundadas en principios intersubjetivos justificados, o sea, que sean el producto de preferencias impersonales válidas.

De este modo, el reconocimiento de necesidades básicas como dato moral relevante para la distribución igualitaria sirve de escudo de protección del individuo frente a las decisiones y preferencias de otros.

Esto permite mostrar que hay una relación entre el principio de autonomía personal y el de inviolabilidad de la persona, ya que mientras el primero estipula que la vida de un individuo sólo debe estar afectada por sus preferencias personales, además de 
por accidentes no compensables o por acciones o decisiones fundadas en principios intersubjetivos válidos, el segundo principio proscribe que la vida de un individuo esté afectada por preferencias personales de otros individuos; es obvio que esta segunda idea está implicada por la primera. Esto tal vez muestre que los dos aspectos distintivos del utilitarismo -su carácter agregativo y su concepción del bien como satisfacción de preferencias- tal vez no sean independientes; si las preferencias dieran razones para actuar que dependen de su contenido y alcance, la vida de un individuo estaría tan afectada por sus propias preferencias personales como por las de los demás.

De este modo, el concepto de necesidades básicas no sólo sería central en una concepción liberal de la sociedad, sino que haría de puente -al permitir su satisfacción simultánea- entre las dos ideas básicas del liberalismo: la de que los fines de los individuos deben ser respetados y la de que todo individuo es un fin en sí mismo». ${ }^{13}$

${ }^{13}$ Le agradezco a Carlos Rosenkrantz las discusiones esclarecedoras que hemos tenido sobre los temas tratados en este artículo.

$\triangle \quad$ DOXA 7 (1990) 